\title{
Modelling of Halbach Array Based Targeting Part of a Magnetic Drug Delivery Device
}

\author{
Inga SKIEDRAITE*, Egidijus DRAGASIUS**, Saulius DILIUNAS*** \\ *Kaunas University of Technology, Studentu Str. 56-322, 51424 Kaunas, Lithuania, E-mail: inga.skiedraite@ktu.lt \\ **Kaunas University of Technology, Studentu Str. 56-321, 51424 Kaunas, Lithuania, E-mail: egidijus.dragasius@ktu.lt \\ ***Kaunas University of Technology, Studentu Str.56-342, 51424 Kaunas, Lithuania, E-mail: saulius.diliunas@ktu.lt \\ cross $^{\text {ref }}$ http://dx.doi.org/10.5755/j01.mech.23.6.19645
}

\section{Introduction}

Research on magnetic drug targeting as a therapeutic technique, which requires guidance of drug carriers to specific sites in a manner such that its major fraction interacts exclusively with a target tissue at the cellular or subcellular level is receiving more and more attention [1-3]. Typically, the intended drug and a suitable magnetically active component are formulated into a pharmacologically stable compound which is injected through an appropriate blood vessel supplying the targeted zone in the presence of an external magnetic field with sufficient field strength and gradient to retain the carrier particles at the target site. The focused administration of drugs is carried out by use of magnets to direct drug carriers of appropriate size and magnetic properties to specific sites in human body to treat tumours, infections, blood clots, for local hyperthermia and other [49]. Such administration can reduce unwanted distribution of drugs thus minimizing side effects such as caused by systematically administered chemotherapy [10].

Applied magnetic field and magnetic field gradient together with the vascularization of target region are particularly important when considering the distance from the magnets to the locations where particles are still effectively captured [8]. Applied magnetic field and magnetic field gradient decrease rapidly with increase of distance from magnets; therefore, insufficient reach is one of the major limits of the applicability of magnetic drug delivery [2, 6, 11-17]. It is still a technically challenging task to build up sufficient field strength that would sufficiently focus drug carriers on a small area and would be able to counteract the linear blood-flow rates acting on said drug carriers in blood vessels $(10 \mathrm{~cm} / \mathrm{s}$ in arteries and $0.05 \mathrm{~cm} / \mathrm{s}$ in capillaries [1].

It is a common practice to use basic shape permanent magnets or electromagnets to pull carrier particles into a target tissue by placing said magnets in close proximity to the target zone for accumulation of magnetic drug carriers at a predetermined site. It was reported that strength of used magnets and magnetic field gradient varies from $70 \mathrm{mT}$ to $2.2 \mathrm{~T}$ and from $0.03 \mathrm{~T} / \mathrm{m}$ to $100 \mathrm{~T} / \mathrm{m}$, respectively $[3,9,12$, 18]. To date in human clinical trials $[1,11]$ using $100 \mathrm{~nm}$ diameter particles a focusing depth of $0.5 \mathrm{~cm}$ has been achieved. In experiments with animals [18] reported targeting depth was up to $12 \mathrm{~cm}$ using $0.2-0.8 \mathrm{~T}$ strength magnets and $0.5 \mu \mathrm{m}-5 \mu \mathrm{m}$ magnetic particles. Restricted treatment depths and thus focusing accuracy of drug carriers mean that only a fraction of patients could be treated with magnetic drug delivery.

Although most active magnetic drug targeting sys- tems are based on pull forces generated by a single permanent magnet placed near the target tissue use of superconducting magnets capable of producing high magnetic fields has been suggested as a way of reaching deeper locations in the body. Nevertheless, under conditions of magnetically non-saturated particles the magnetic force on the particles is proportional to both the external magnetic field and the magnetic field gradient. With increasing external magnetic field, the particle eventually will reach magnetic saturation thereafter the magnetic force will be proportional only to the magnetic field gradient [6]. By increasing dimensions of magnets generated field tends to become homogeneous over the target site, resulting in a small field gradient. Hence, increasing the magnetic field by applying a stronger permanent magnet will not necessarily increase the magnetic force on a magnetic particle. There are reports on using magnetic implants within a target zone in blood vessels as an alternate way to increase magnetic drug targeting strength in deep tissues [19]. The implanted material serves to locally increase the magnetic field gradients when an external magnetic field is applied. Nevertheless, this method also lacks solid research data on its effectiveness.

Attention in this paper has been focused to the issue of lack of sufficiently large magnetic field gradient at a certain distance from its source.

\section{Magnet arrangement of magnetic targeting devise based on Halbach array}

In principle the magnetic force on a magnetic particle can be described as [3]

$$
\overrightarrow{F_{m}}=\frac{4 \pi a^{3}}{3} \cdot \frac{\mu_{0} \chi}{1+\chi / 3}\left(\frac{\partial \vec{H}}{\partial \vec{\chi}}\right) \vec{H}=\frac{2 \pi a^{3}}{3} \cdot \frac{\mu_{0} \chi}{1+\chi / 3} \nabla(\vec{H})^{2}
$$

where $\vec{H}$ is the magnetic field flux density, $\mathrm{A} / \mathrm{m}, \chi$ is the magnetic susceptibility, and $\mu_{0}=4 \pi \cdot 10^{-7}$ is the permeability of vacuum, a is the radius of the particle, $m, \nabla$ is the gradient operator, $1 / \mathrm{m}$. The first relation shows that a partially varying magnetic field $\left(\frac{\partial \vec{H}}{\partial \vec{\chi}}\right) \neq 0$ is required to create magnetic forces. It also shows that the force on a single particle is directly proportional to its volume. The second relation, which is equivalent to the first one, states that the force on particles is along the gradient of the magnetic field intensity squared - i.e. ferromagnetic particle will always experience a force from low to high applied magnetic field. 
In other words, for magnetic particles to be diverted from their path after injection into blood stream and afterwards to be accumulated at a target zone the drag force $\overrightarrow{F_{t}}$ has to gradually decrease and become smaller than the magnetic force $\overrightarrow{F_{m}}$ when magnetic particles are approaching target zone

$$
\overrightarrow{F_{t}}=6 \pi r(\vec{v}-\vec{u})<\overrightarrow{F_{m}}=\frac{2 \pi a^{3}}{3} \cdot \frac{\mu_{0} \chi}{1+\chi / 3} \nabla(\vec{H})^{2},
$$

where $\vec{v}$ is the velocity of a particle $(\mathrm{m} / \mathrm{s}), \vec{u}$ is a velocity of a carrier fluid, $\mathrm{m} / \mathrm{s}, r$ is radius of a particle, $\mathrm{m}$.

Because the goal is to increase deep reach distance for control of drug carrying magnetic particles by means of permanent magnets, Halbach arrays [13, 20-22] composed of permanent rectangular sub-magnets arranged in a linear manner (Fig. 1) were used.

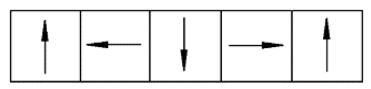

Fig. 1 General representation of permanent magnets arranged in Halbach array for one wavelength

Here two independent variables are considered: a number of magnets per wavelength and total number of

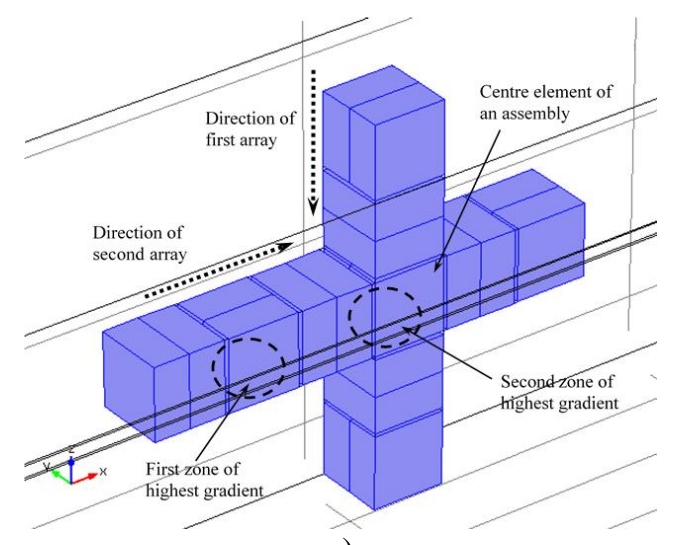

a) wavelengths in an array. As number of wavelengths increases, the magnetic field of each array becomes stronger but the magnetic field lines exhibit smaller extrusions outside the magnetic array before returning. Thus, the force becomes stronger but over a smaller distance. If the strength of a Halbach array is unrestricted, then the magnetic force can be increased simply by using stronger magnets. However, there are practical constraints on the available strengths of permanent magnets as well as regulatory safety constraints on the strength of the magnetic field that can be applied across the human body.

A Halbach array based assembly (Fig. 2) was formed from rectangular permanent magnets, which were arranged to form a cross-like structure. In this structure: two permanent magnets are forming a single direction magnetized element and where each of said elements is in relation to every consecutive element so that a two linear Halbach arrays would be formed in a perpendicular one to another manner having one common member at the centre of the assembly (Fig. 2, a).

So far, Halbach arrays have been implemented only for near surface magnetic focusing [13, 23], but not for deep reach. The aim is to investigate the magnetic drug delivery device, in particular to design a targeting part of magnetic drug targeting device based on Halbach arrays, where the device has two separate blocks where one block can be movable with respect to another one (Fig. 2, b).

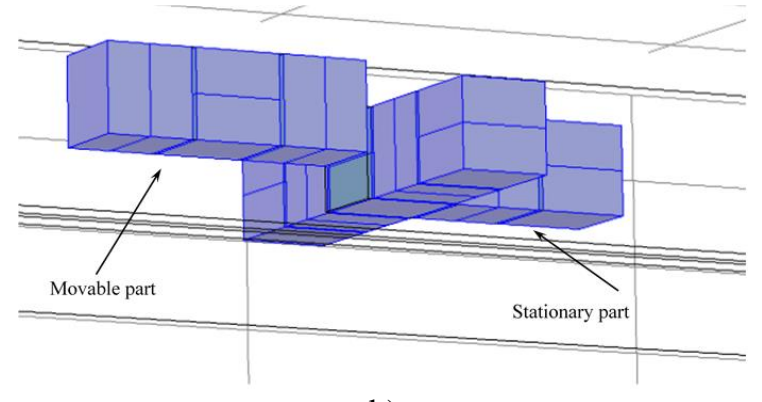

b)

Fig. 2 Configuration of magnet arrangement: a) straight; b) lifted

\section{Method of steering magnetic carriers}

When particles are injected into a stream of liquid they tend to disperse at the moment of injection and only particles that are captured by the fast flowing layers of the middle zone are carried downstream. In case of blood vessels, the lumen is defined by rough surface of endothelium layer providing means for immobilizing particles at the site of interaction. The model is based on assumption, that magnetic drug carriers arriving at the zone of target in the fast flowing regions of blood stream together with major fraction of red blood cells and other blood constituents. In order to facilitate capturing of magnetic carriers, instead of a MDT (magnetic drug targeting) device with single point of highest magnetic field gradient assembly from magnets of MDT device containing two points of highest magnetic field gradient was designed (Fig. 2, a). First zone of highest magnetic field gradient serves as a diverting means for diverting magnetic carriers from the fast flowing region of a blood stream to the slower regions containing significantly fewer red blood cells, closer to a wall of a blood vessel. Moreover, in order to have only one zone of aggregation of particles, i.e. only at the target area, part of the assembly from magnets, forming the diverting magnetic field gradient zone, is lifted from the common plane of the assembly. In such a manner, the second zone of highest magnetic field gradient becomes dominant and produces pulling force on the magnetic carriers sufficient to guide them from the slower blood stream region to the targeting point and immobilize them thereat.

In addition, previous experiment led to conclusion that it is more efficient in terms of captured particles to supply certain quantity of particles at a higher input flow rate than the same quantity at a lower rate. This is due to the interaction of magnetic field and particles: lower concentration of particles per certain volume of flowing liquid gives weaker magnetic interaction between particles thus weaker 
overall effect of the magnetic field gradient to said particles. Greater concentration allows faster formation of an anchor body (bulk of suspended particles) whereat the magnetic force of the anchor attracts the inlet particles causing their velocity to increase. It has been determined both experimentally and theoretically that particles moving faster than local shear flow will experience additional forces toward the walls of a vessel [12].

A problem of optimal assembly from magnets modelling for steering of magnetic nanoparticles in a closed environment containing a stream of close-to-blood liquid is considered in this research. The targeting part of a device was designed based on Halbach arrays and contains 18 permanent magnets having 20x20x10 mm dimensions, which form a movable, and a stationary block of the targeting part of a magnetic drug delivery device for use in magnetic drug targeting therapy.

Main problem in such technique is rapidly diminishing magnetic field together with magnetic field gradient and subsequently magnetic field force, which drives magnetic nanoparticles towards the targeting area. For particles to be successfully manipulated in a blood stream to the area of immobilization the magnetic device has to produce such magnetic field gradient. In other words, the particles have to be diverted from fast moving area of blood stream in a blood vessel to slow moving section so that bloodstream velocity $\vec{v}$ would be less or equal to velocity of a particle $\vec{u}$ (Fig. 3 ).

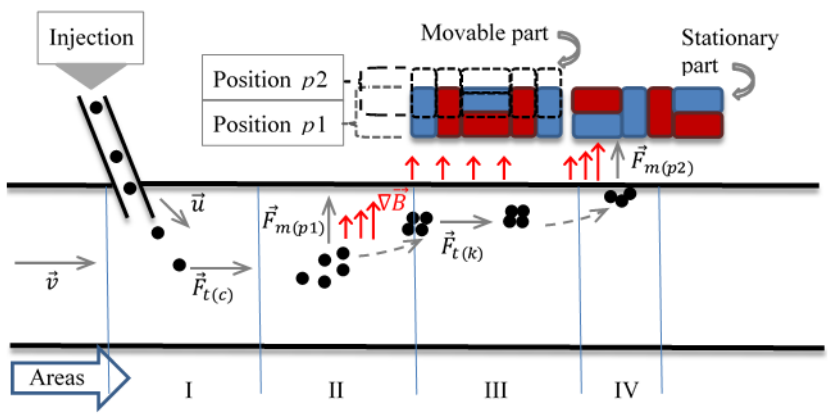

Fig. 3 Magnetic particle motion trajectory under the targeting blocks

Magnetic particles together with drug are injected in the area I, where they are affected by a force $\overrightarrow{F_{t(c)}}$, which is formed by the blood stream. The particles are injected in the area I at some distance from the magnetic device that they would not be exposed to the generated magnetic field and will not start to move towards the blood vessel wall too early and to avoid formation of magnetic particles aggregation.

In particle steering scheme in Fig. 3 in area II particles experience magnetic field pulling force $\overrightarrow{F_{m(p 1)}}$, which is achieved when the assembly is in straight configuration and movable element of the assembly is in position $\mathrm{p} 1$. When the particles reach front edge of the assembly (at the division line between area I and are II) they are in slower flowing region in the blood vessel and subsequently $\overrightarrow{F_{t(k)}}$ becomes only slightly stronger then $\overrightarrow{F_{m(p 1)}}$. At this point, when the particles are in area III the movable part of the assembly is moved to position $p 2$. The drag force $\overrightarrow{F_{t(k)}}$ be- comes equal or less then $\overrightarrow{F_{m(p 1)}}$ and particles start moving at a declined angle trajectory to the area IV, where $\overline{F_{m(p 2)}}$ becomes essentially stronger then $\overrightarrow{F_{t(k)}}$, and thus particles are aggregated at that area beneath the central element of the assembly from magnets.

\section{Model of magnetic field distribution}

In order to assess particle trajectory under the influence of magnetic field gradient $\nabla(\vec{B})^{2}$, which is generated by the targeting blocks of a MDT device the finite element software COMSOL Multyphysics environment was used. The target was to model such configuration of the assembly from magnets which would force magnetic nanoparticles to change their flow course in the blood stream (areas I and II, Fig. 3) and migrate to area where blood flow velocity is reduced (area III, Fig. 3). The movable block acts as a diverter and is moved to position $p 2$ (Fig. 3) so the flowing particles would not accumulate below the concentration area of said movable block and would be free to reach the target area under the middle section of the stationary block (area IV, Fig. 3) where particles are accumulated.

Model was constructed using eighteen NdFeB N42 class permanent magnets with remnant magnetization of 1.3 T. The fluid flow was modelled in a $2 \mathrm{~mm}$ wide and $300 \mathrm{~mm}$ long glass tube. The flowing liquid had dynamic viscosity of $3 \cdot 10^{-3} \mathrm{~Pa} \cdot \mathrm{s}$ (or $\mathrm{N} \cdot \mathrm{s} / \mathrm{m}^{2}$ ). Around 1000 nanoparticles having $500 \mathrm{~nm}$ diameter where injected upstream of the device at about $170 \mathrm{~mm}$ distance from the edge of the movable block. The boundary conditions: the magnetic field

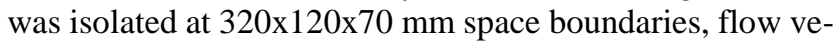
locity at the channel walls was $0 \mathrm{~m} / \mathrm{s}$, the velocity at the inlet was $0.1 \mathrm{~m} / \mathrm{s}$, particles bounce from the wall in tangential direction. The modelling was done in combined environment of magnetic (no currents) module, fluid flow module and particle traction in fluid module.

Figs. 4 to 6 give a comprehensive representation of magnetic field spatial distribution below the assembly from magnets. Two models are presented each being in straight and lifted configurations and one model in straight configuration only.

In model 1 (Fig. 4), straight configuration the distance between the assembly surface and the fluid flow channel is $9 \mathrm{~mm}$ where magnetic field distribution has two major peaks and when in lifted configuration the distance between the lifted part of the assembly and the fluid channel is $19 \mathrm{~mm}$ where magnetic field distribution has one major peak. In both cases, value of the central peak stays the same. It can be seen that bellow the common part of two intersecting linear Halbach arrays magnetic field flux extends furthest, thus providing deeper control of magnetic nanoparticles.

In case of model 2 (Fig. 5), the distance between assembly from magnets surface and the fluid flow channel is $19 \mathrm{~mm}$, where magnetic field distribution has one distinctive peak. Moreover, when in lifted configuration the distance between the lifted part of the assembly and the fluid channel is $29 \mathrm{~mm}$, where magnetic field distribution major peak value is slightly decreased. 


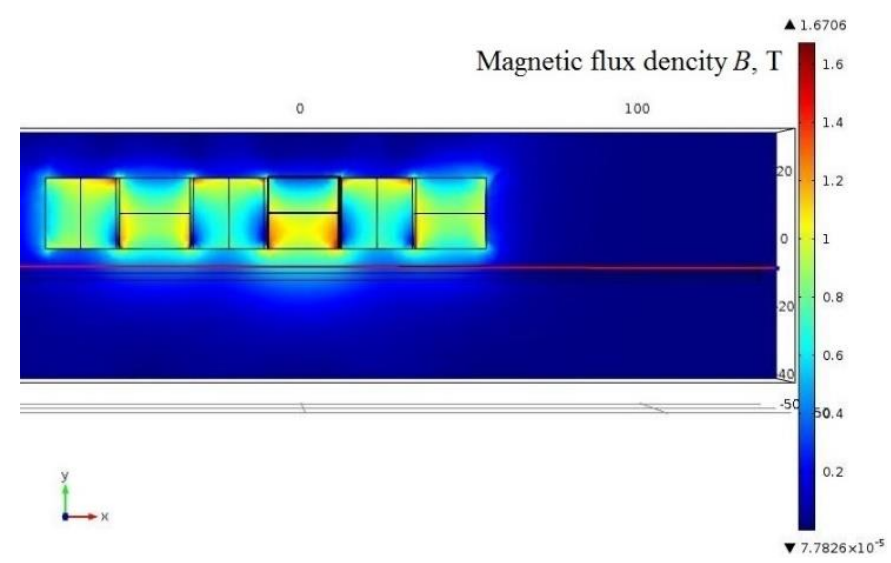

a)

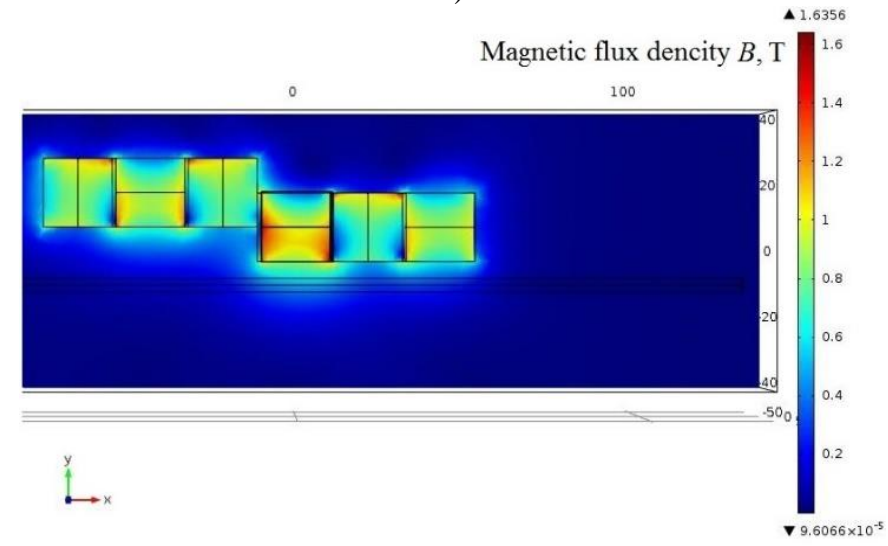

c)

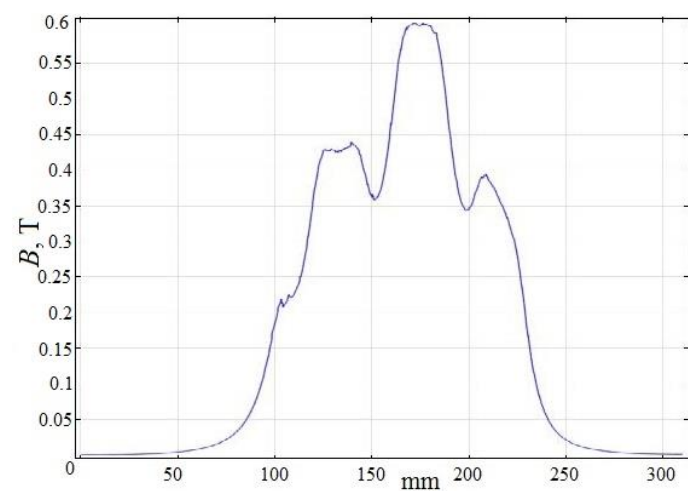

b)

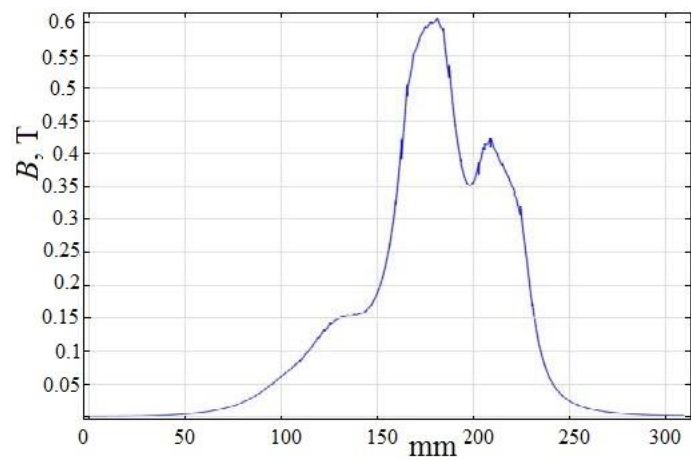

d)

Fig. 4 Model 1: a) straight configuration; b) magnetic field distribution of straight configuration; c) lifted configuration; d) magnetic field distribution of lifted configuration
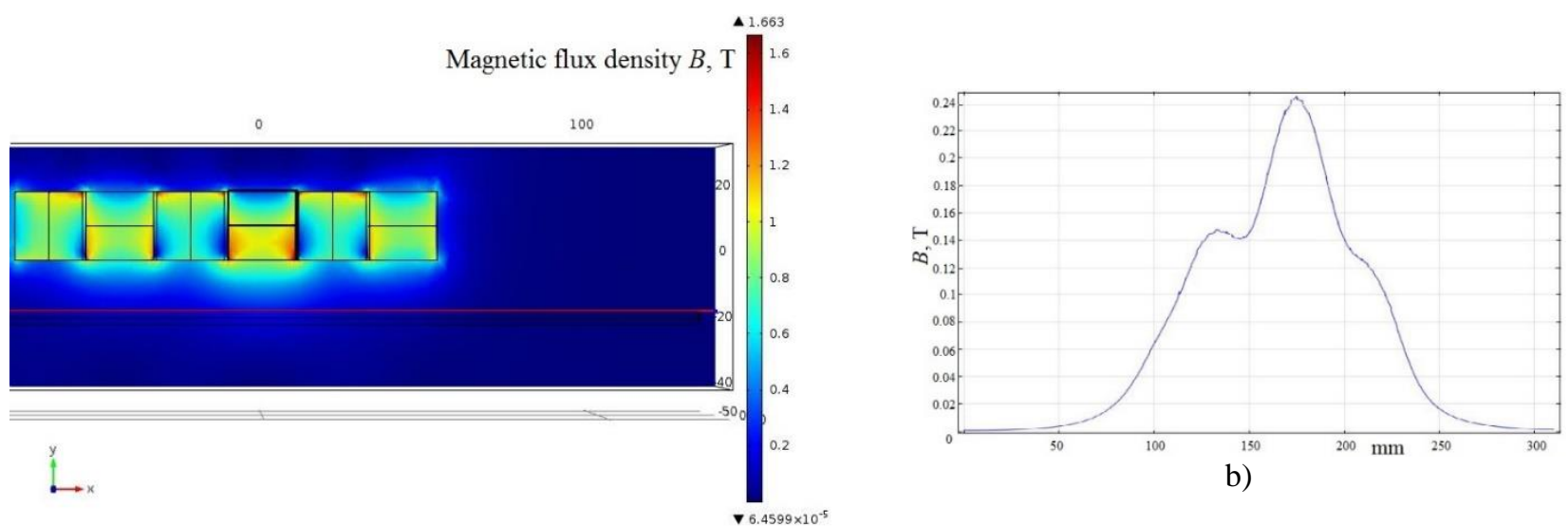

b)
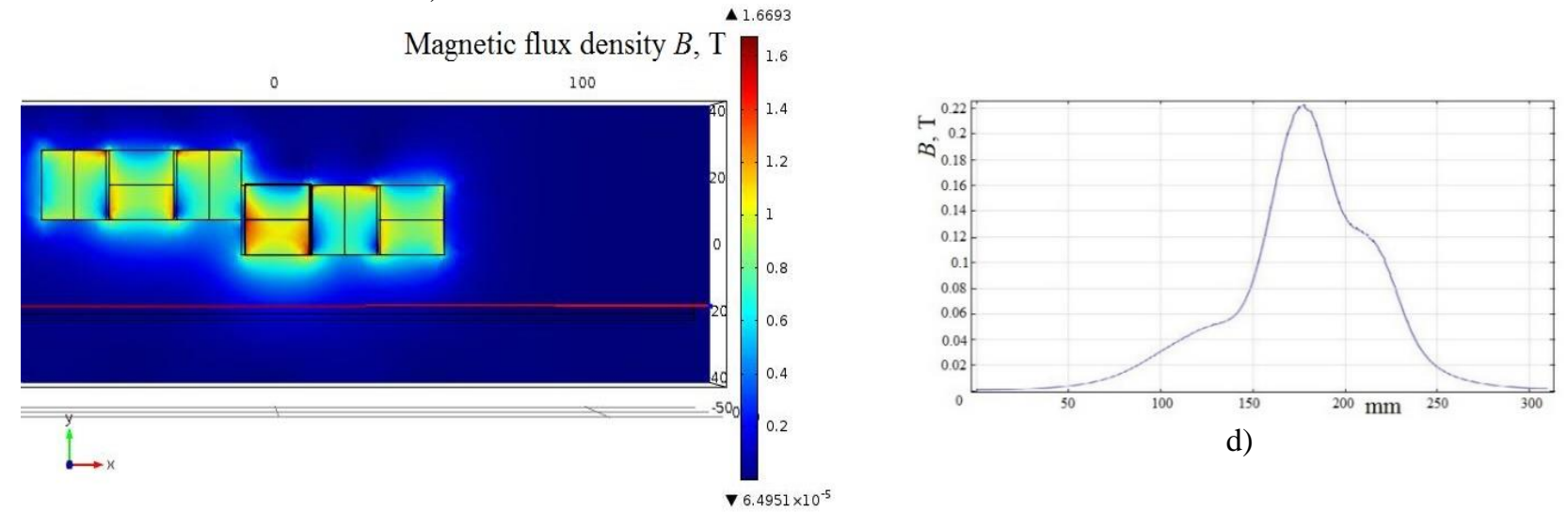

d)

c)

Fig. 5 Model 2: a) straight configuration; b) magnetic field distribution of straight configuration; c) lifted configuration; d) magnetic field distribution of lifted configuration 
Model 3 (Fig. 6) represents conditions when particles do not experience enough magnetic field force and are flushed away: here distance between the assembly from magnets surface in straight configuration and the fluid flow channel is $19 \mathrm{~mm}$. By working through numerous models of presented assembly deepest reach of magnetic field was confirmed with present configuration of permanent magnets.

\section{Trajectories of magnetic particles and acting forces}

In order to analyse the effect of the assembly from magnets formed magnetic field to the flowing magnetic nanoparticles under the assembly, laminar fluid flow and particle tracking physics were combined. First model (Fig. 7, a, b) was built in straight assembly configuration (Fig. 4). Distance between the assembly and the surface of the channel was $9 \mathrm{~mm}$ throughout the length of the assembly. The particles changed their trajectory after $0.8 \mathrm{~s}$ after being released from the inlet, which was $170 \mathrm{~mm}$ away from the front part of the firs magnet of the assembly. The particles didn't change their flow path in the time frame from $5 \mathrm{~s}$ to $60 \mathrm{~s}$ (modelling time). First model in lifted configuration (Fig. 7, $\mathrm{c}, \mathrm{d}$ ) contained a lifted part of the assembly from magnets forming a gap between the assembly and the surface of the channel is of $19 \mathrm{~mm}$ where the unmoved part remained at $9 \mathrm{~mm}$ distance from the channel. The magnetic particles changed their trajectory after 1 second after release from the inlet and in the time period from $6 \mathrm{~s}$ to $60 \mathrm{~s}$ of the modelling time the flow paths stayed the same. The colour in graph represents velocity $\mathrm{m} / \mathrm{s}$ of particles.
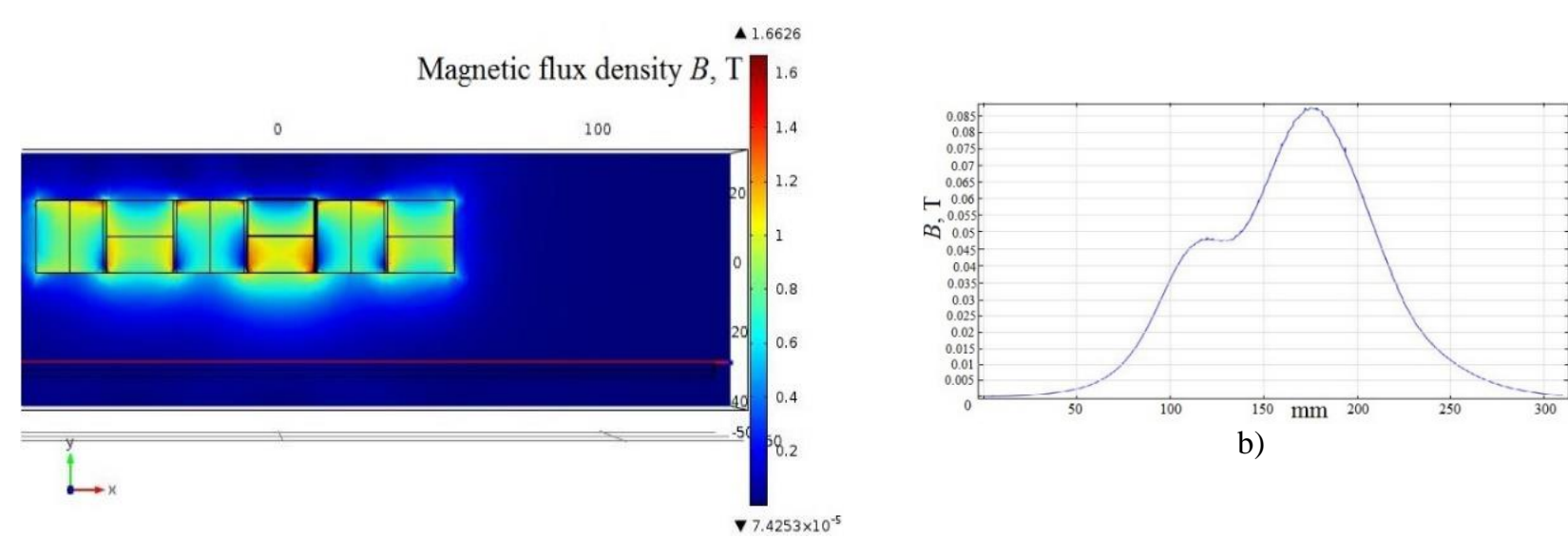

b)

a)

Fig. 6 Model 3: a) straight configuration; b) magnetic field distribution

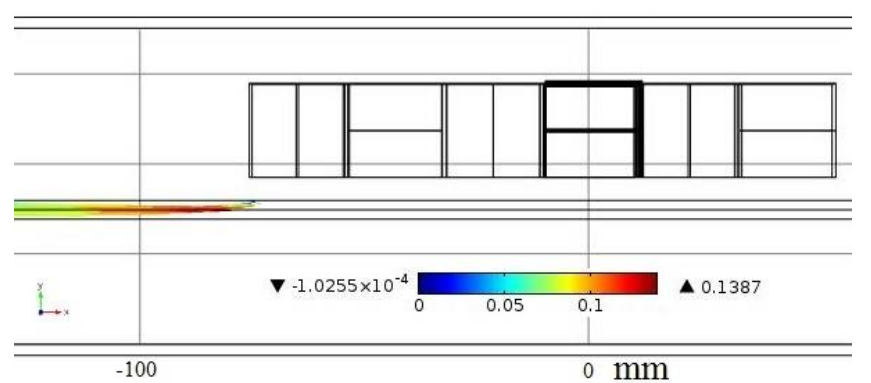

a)

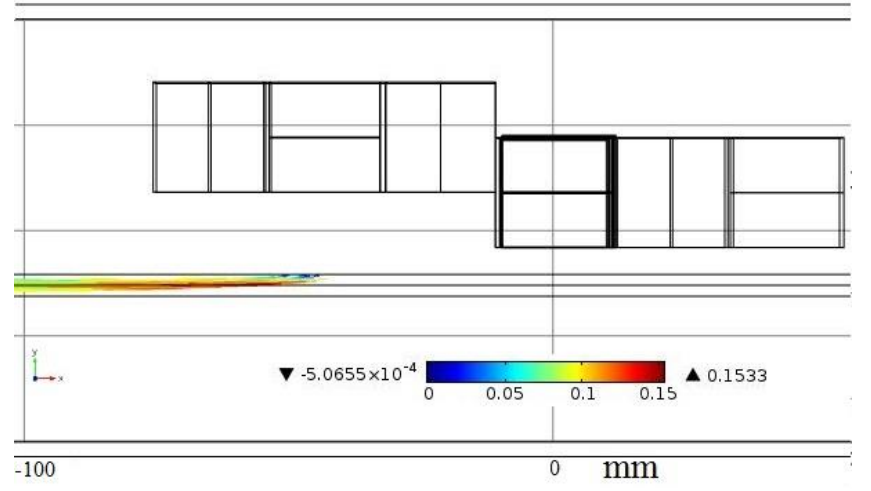

c)

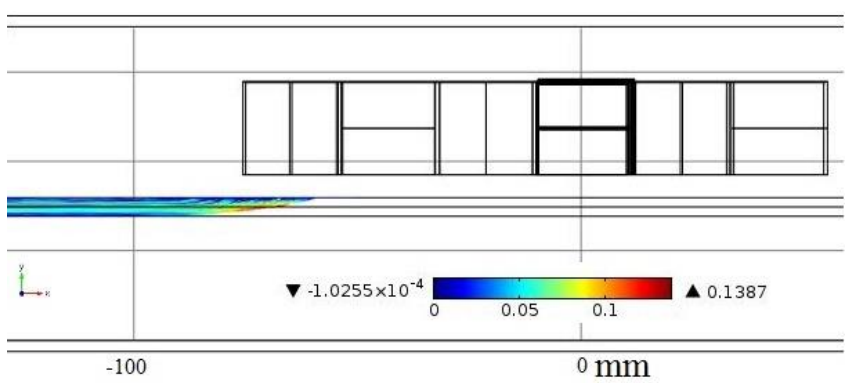

b)

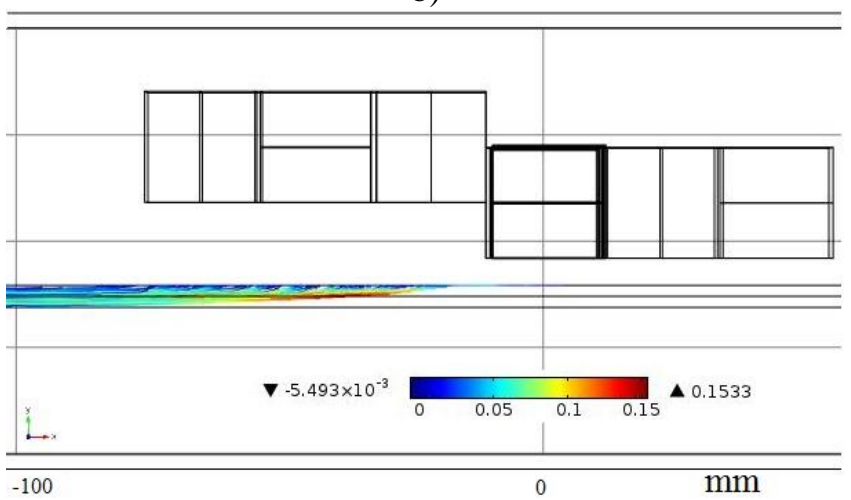

d)

Fig. 7 Magnetic nanoparticle trajectories of first model after release from the inlet: a) straight configuration - after $5 \mathrm{~s}$; b) straight configuration - after $60 \mathrm{~s}$; c) lifted configuration - after $6 \mathrm{~s}$; d) lifted configuration - after $60 \mathrm{~s}$

Second model (Fig. 8) was configured in the same manner as the previous one except the distance in both straight (Fig. 8, a, b) and lifted (Fig. 8, c, d) configurations was increased by $10 \mathrm{~mm}$. The particles changed their trajectory after 1 second and the flow path trajectories remained unchanged from $6 \mathrm{~s}$ to $60 \mathrm{~s}$ of the modelling time in straight 
configuration of the assembly from magnets. In lifted configuration of the assembly the particles changed their trajectory after $1.3 \mathrm{~s}$. The particles did not change their flow path in the period from $7 \mathrm{~s}$ to $60 \mathrm{~s}$ of the modelling time.

In the first model in straight configuration after $0.8 \mathrm{~s}$ of injection, the injected particles change their motion path towards the assembly from magnets and start moving

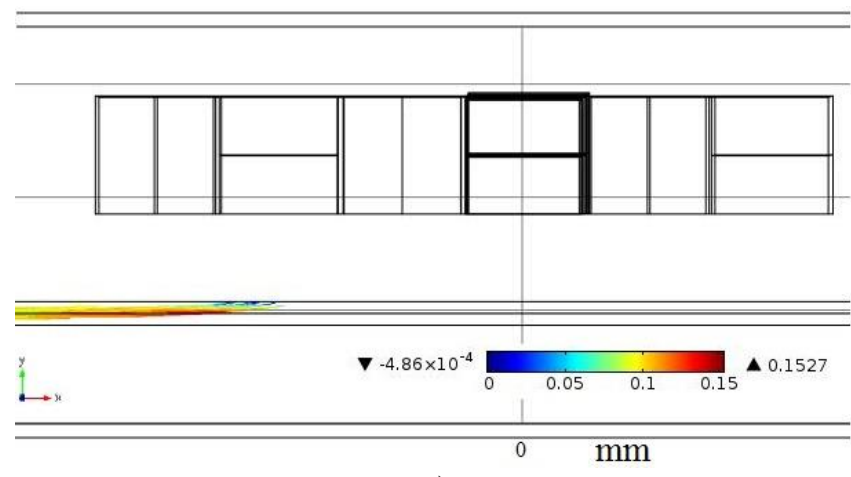

a)

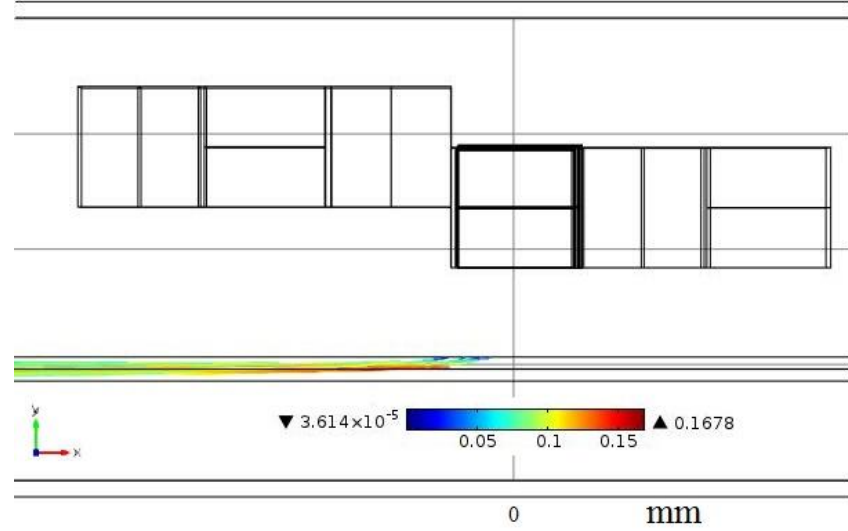

c) faster than the velocity of the surrounding liquid layer. The drag force acting on particles is in the range from $-30 \times 10$ $16 \mathrm{~N}$ to $-15 \times 10-16 \mathrm{~N}$ (Fig. 9, a, b). The minimum magnetic force on particles, necessary to divert particles from their path is in the range from $4 \times 10-12 \mathrm{~N}$ to $1.6 \times 10-12 \mathrm{~N}$ (Fig. 9, c, d).

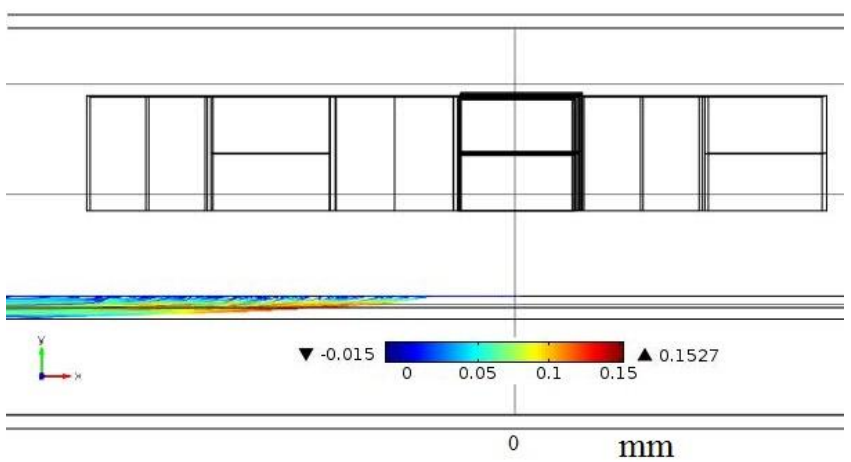

b)

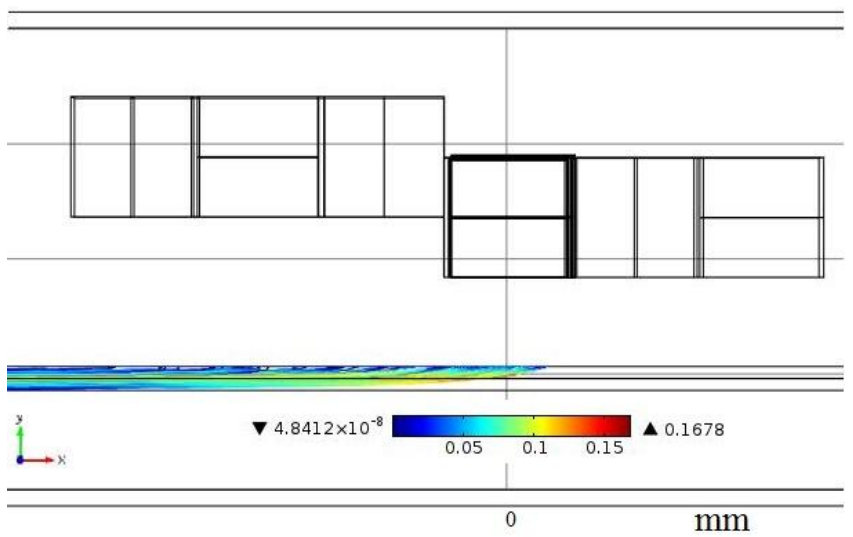

d)

Fig. 8 Magnetic nanoparticle trajectories of second model after release from the inlet: a) straight configuration - after $5 \mathrm{~s}$;

b) straight configuration - after $60 \mathrm{~s}$; c) lifted configuration - after $6 \mathrm{~s}$; d) lifted configuration - after $60 \mathrm{~s}$

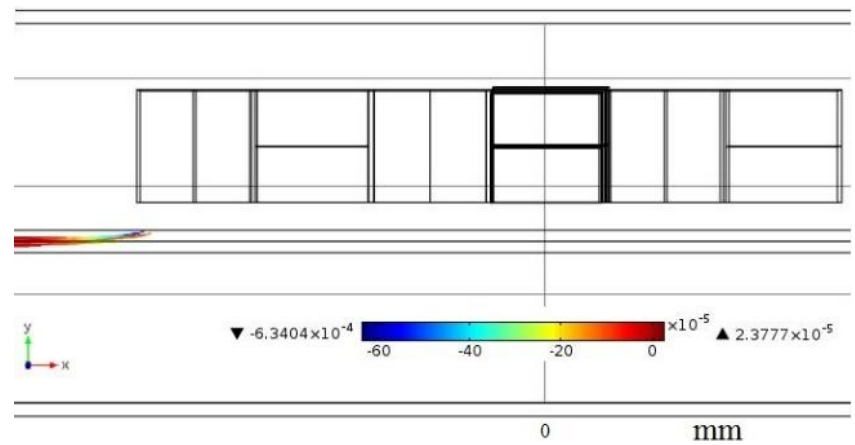

a)

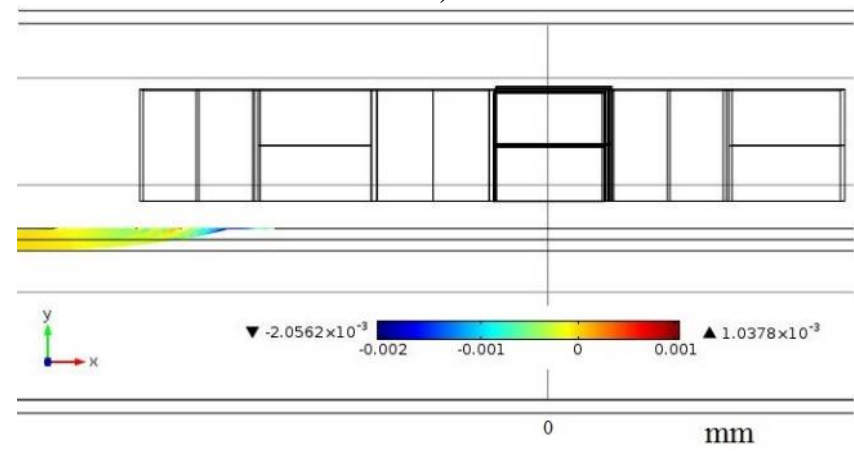

c)

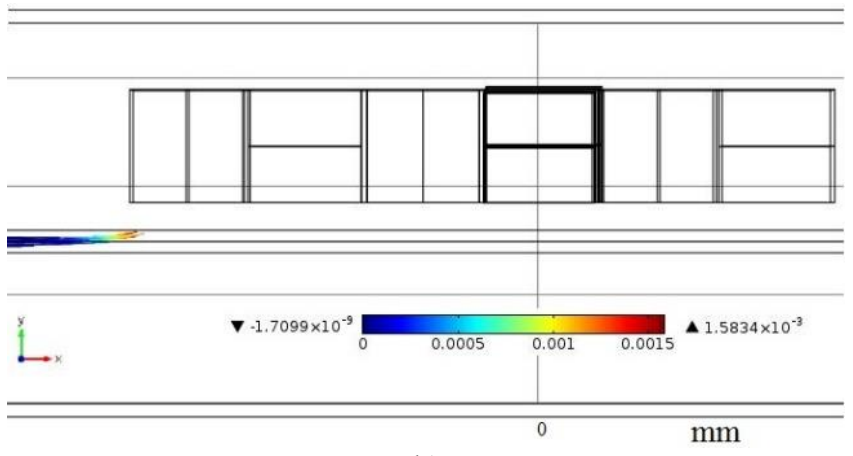

b)

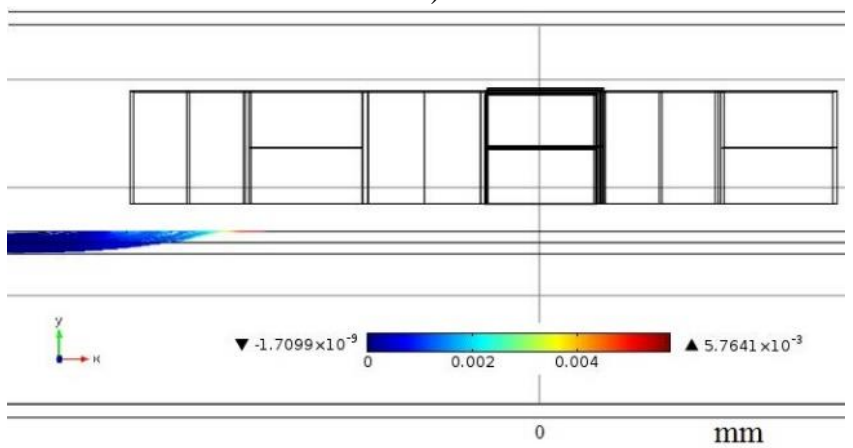

d)

Fig. 9 Forces acting on particles in first model: a) drag force after $0.8 \mathrm{~s}$; b) magnetic force after $0.8 \mathrm{~s}$; c) drag force after $60 \mathrm{~s}$; d) magnetic force after $60 \mathrm{~s}$ 


\section{Conclusions}

Main research idea was to investigate the assembly from magnets for magnetic drug targeting (MDT) device and whether the device generates sufficient magnetic field force on particles for them to be able to compete with convective blood (drag) force that tend to wash particles away. Research showed that using special order of permanent magnets it is possible to create magnetic field gradient sufficient enough to trap a magnetic particles having diameter of $500 \mathrm{~nm}$ at a specific location. The targeting distance was only about $19 \mathrm{~mm}$, but it has been reported that $5 \mathrm{~mm}$ is normal achievable distance for targeting research so far. Compared to previous research produced magnetic field of used magnetic device as well as reached gradient values was well in the range of applicable means for magnetic drug targeting.

Moreover, the usage of special ordered magnets with relatively smaller dimensions (for composing a Halbach array type assembly) is more advantageous than using relatively large permanent magnets. Even if it means increase in size of magnets means increase in generated magnetic field values further from surface of a magnet, but the usage of larger magnets to extend the magnetic field to deeper regions may not resolve the problem since low magnetic field gradients tend to result. The particle trajectory and aggregation at the point of interest allow concluding that device is well suited for magnetic drug targeting application, because major amount of injected particles was retained within the area of targeting.

It was shown that particles are diverted to the target area further away from the assembly using modelled configuration of permanent magnets assembly than in case of straight configuration. Depending on the depth of the targeting area under the assembly (within this case was up to 20 $\mathrm{mm}$ ) the assembly may be used in two modes, where one mode requires reciprocating movement of first block of the assembly and another mode is when the assembly stays in straight configuration the whole capturing period. Further minimum magnetic and drag force values where established for particle path manipulation towards the target area.

\section{Acknowledgment}

Authors would like to say thank you to Prof. Bronius Baksys and Mr. Karolis Sileika for their help and input to prepare this paper.

\section{References}

1. Lubbe, A. S.; Alexiou, C.; Bergemann, C. 2001. Clinical Applications of Magnetic Drug Targeting J Surg Res. 95: 200-206. http://dx.doi:10.1006/jsre.2000.6030.

2. Jurgons, R.; Seliger, C.; Hilpert, A.; Trahms, L.; Odenbach, S.; Alexiou, C. 2006. Drug Loaded Magnetic Nanoparticles for Cancer Therapy. J. Phys.: Condens. Matter. 18(38): S2893-S2902. http://dx.doi:10.1088/0953-8984/18/38/S24.

3. Taylor, E.N.; Webster, T.J. 2011. Multifunctional Magnetic Nanoparticles for Orthopedic and Biofilm Infections. Int. J. Nanotechnol. 8(1/2): 21-35. http://dx.doi:10.1504/IJNT. 2011.037168.

4. Kempe, M.; Kempe, H.; Snowball, I.F.; Wallen, R.;
Arza, C.R.; Gotberg, M.; Olsson T. 2010. The Use of Magnetite Nanoparticles for Implant-assisted Magnetic Drug Targeting in Thrombolytic Therapy. Biomaterials 31(36): 9499-9510. http://dx.doi:10.1016/ j.biomaterials.2010.07.107.

5. Jordan, A.; Scholz, R.; Maier-Hauff, K.; van Landeghem, F. K.; Waldoefner, N.; Teichgraeber, U.; Pinkernelle, J.; Bruhn, H.; Neumann, F.; Thiesen, B.; von Deimling A.; Felix R. 2006. The Effect of Thermotherapy Using Magnetic Nanoparticles on Rat Malignant Glioma, JNO 78(1), 29: 7-14.

http://dx.doi: 10.1007/s11060-005-9059-z.

6. Schuell, B.; Gruenberger, T.; Kornek, G.V.; Dworan, N., Depisch, D.; Lang, F.; Schneeweiss, B.; Scheithauer, W. 2005. Side Effects during Chemotherapy Predict Tumour Response in Advanced Colorectal Cancer. BJC 93(7): 744-748. http://dx.doi:10.1038/sj.bjc.6602783.

7. Chertok, B.; Chertoka, B.; Moffatb, B.A.; Davida, A. E.; Yua,F.; Bergemannc, C.; Rossb, B. D.; Yanget, V. C. 2008. Iron Oxide Nanoparticles as a Drug Delivery Vehicle for MRI Monitored Magnetic Targeting of Brain Tumors. Biomaterials 29(4): 487-496. http://dx.doi: 10.1016/j.biomaterials.2007.08.050.

8. Nacev, A.; Beni, C.; Bruno, O.; Shapiro, B. 2011. The Behaviors of Ferromagnetic Nano-particles in and Around Blood Vessels under Applied Magnetic Fields. J. Magn. Magn. Mater. 323(6): 651-668. http://dx.doi:10.1016/j.jmmm.2010.09.008.

9. Ally, J.; Martin, B.; Behrad Khamesee, M.; Roa, W.; Amirfazli, A. 2005. Magnetic Targeting of Aerosol Particles for Cancer Therapy. J. Magn. Magn. Mater. 293(1): 442-449. http://dx.doi:10.1016/j.jmmm.2005.02.038.

10. Takeda, S.; Mishima, F.; Fujimoto, S.; Izumi, Y.; Nishijima, S. 2007. Development of Magnetically Targeted Drug Delivery System Using Superconducting Magnet. J. Magn. Magn. Mater. 311(1): 367-371.

http://dx.doi:10.1016/j.jmmm.2006.10.1195.

11. Agotegaray, M. A.; Lassalle, V. L. 2017. Silica-coated Magnetic Nanoparticles. An Insight into Targeted Drug Delivery and Toxicology. Springer, Swiztzerland.

12. Forbes, Z. G.; Yellen, B. B.; Barbe,e K. A.; Friedman, G. 2003. An Approach to Targeted Drug Delivery Based on Uniform Magnetic Fields. IEEE Trans. Magn. 39(5): 3372-3377. http://dx.doi: 10.1109/TMAG.2003.816260.

13. Hayden, M. E.; Hafeli, U. O. 2006. 'Magnetic Bandages' for Targeted Delivery of Therapeutic Agents. J. Phys.: Condens. Matter. 18(38): S2877-S2891. http://dx.doi:10.1088/0953-8984/18/38/S23.

14. Zheng, J.; Wang, J.; Tang, T.; Li, G.; Cheng, H.; Zou, S. 2006. Experimental Study on Magnetic Drug Targeting in Treating Cholangiocarcinoma Based on Internal Magnetic Fields. Chin Ger J Clin Oncol. 5(5): 336-338. http://dx.doi:10.1007/s10330-006-0492-z.

15. Gosiewski, Z.; Mystkowski, A. 2008. Robust control of active magnetic suspension: analytical and experimental results. Mech Syst Signal Process 22(6): 1297-1303. http://dx.doi:10.1016/j.ymssp.2007.08.005.

16. Mystkowski, A. 2011. An application of mu-synthesis to control of a small air vehicle - simulation results. J. Vibroeng. 14(1): 79-86. 
17. Mystkowski, A.; Jastrzębski, R. 2013. Vibrating a small plate vortex generator to improve control robustness of a micro aerial delta wing vehicle. J. Vibroeng. 15(1): 114-123.

18. Goodwin, S.; Peterson, C.; Hoh, C.; Bittner, C. 1999. Targeting and Retention of Magnetic Targeted Carriers (MTCs) Enhancing Intra-arterial Chemotherapy. J. Magn. Magn. Mater. 194(1-3): 132-139. http://dx.doi:10.1016/S0304-8853(98)00584-8.

19. Forbes, Z. G.; Yellen, B. B.; Halverson, D. S.; Fridman, G.; Barbee, K.A.; Friedman, G. 2008. Validation of High Gradient Magnetic Field Based Drug Delivery to Magnetizable Implants Under Flow. IEEE Trans. Biomed. Eng. 55(2): 643-649. http://dx.doi:10.1109/TBME. 2007.899347.

20. Robertson, W.; Cazzolato, B.; Zander, A. 2010. Parameters for Optimizing the Forces between Linear Multipole Magnet Arrays. IEEE Magn. Lett. 1. http://dx.doi:10.1109/LMAG. 2010.2047716.

21. Hafeli, U.O.; Gilmour, K.; Zhou, A.; Lee, S.; Hayden, M. E. 2007. Modeling of Magnetic Bandages for Drug Targeting: Button vs. Halbach Arrays. J. Magn. Magn. Mater. 311(1): 323-329.

http://dx.doi:10.1016/j.jmmm.2006.10.1152.

22. Raich, H.; Blumler, P. 2004. Design and Construction of a Dipolar Halbach Array with a Homogeneous Field from Identical Bar Magnets: NMR Mandhalas. Concepts Magn Reson: Part B: Magnetic Resonance Engineering 23B(1): 16-25. http://dx.doi:10.1002/ cmr.b.20018.

23. Holligan, D.L.; Gillies, G.T.; Dailey, J.P. 2003. Magnetic Guidance of Ferrofluidic Nanoparticles in an in Vitro Model of Intraocular Retinal Repair. Nanotechnology 14(6): 661-666.

http://dx.doi:10.1088/0957-4484/14/6/318.
Inga SKIEDRAITE, Egidijus DRAGASIUS, Saulius DILIUNAS

\section{MODELLING OF HALBACH ARRAY BASED \\ TARGETING PART OF A MAGNETIC DRUG DELIVERY DEVICE}

S u m m a r y

A problem of optimal assembly from magnets modelling for steering of magnetic nanoparticles in a closed environment containing a stream of close-to-blood liquid is considered in this research. The targeting assembly from magnets is designed based on Halbach arrays and contains 18 permanent magnets forming a movable and a stationary block of the targeting part of a magnetic drug delivery device. The injected particles were diverted from their flow path near the vicinity of movable part of assembly from magnets while not aggregating thereat, but migrating to the target zone under the stationary part of the said assembly. Minimum magnetic force value was established for the injected particle path manipulation towards the target area.

Keywords: magnetic drug targeting (MDT), control of magnetic particles, Halbach array, magnetic field distribution.

Received September 03, 2017 Accepted December 07, 2017 